Even if centrifugation does not cause aggregation, completely unaggregated preparations could be expected only from plants that have been recently infected, for aggregation occurs naturally in the sap of plants that have been long infected ${ }^{4}$.

$W_{\theta}$ have emphasized the need for caution in interpreting centrifugal data on systems which can, in some circumstances, aggregate. It may be possible to isolate unaggregated tobacco mosaic virus by short periods of centrifuging in strong gravitational fields at a low temperature. But it is significant that four sedimentations cause an increase in anisotropy of flow detectable by the method of Loring, Lauffer and Stanley. It therefore seems improbable that one sedimentation should have no effect. Also, until more adequate data on the activity, chemical composition and physical properties of virus preparations made solely by high-speed centrifugation have been published, it will not be possible to assess their purity.

\footnotetext{
${ }^{\mathrm{t}}$ Bawden, F. C., and Pirie, N. W., Proc. Roy. Soc., B, 123, 309 (1937). ${ }^{2}$ Bawden, F. C., and Pirie, N. W., Reunion Inter. de Physique, Chimie et Biologie (Paris, 1937), p. 382

'Lauffer, M. A., and Stanley, W. M., J. Biol. Chem., 123, 507 (1938).
}

'Wyckoff, R. W. G., J. Biol. Chem., 121, 219 (1937).
DR. Kenneth M. Smith, F.R.S., Potato Virus Research Station, Cambridge, AND W. D. MacClement, Molteno Institute, Cambridge.

DR. Stanley and his colleagues draw the conclusion that tobacco mosaic virus is not aggregating after centrifugation since it passes a membrane of $190 \mathrm{~m} \mu$ average pore diameter. In our ultrafiltration experiments, we have found that this virus, after precipitation at $p \mathrm{H} 3 \cdot 4$, will pass through a membrane of smaller pore size than the foregoing and has an end-point under optimum conditions of 150 $175 \mathrm{~m} \mu$. This indicates an aggregation of some kind, since the accepted ultrafiltration end-point of tobacco mosaic virus in crude clarified sap is about $50 \mathrm{~m} \mu$. In our opinion, therefore, it is not justifiable to draw the conclusion that the virus is not aggregated merely because it passes a membrane of $190 \mathrm{~m} \mu$ average pore diameter. Tobacco mosaic virus, however, which has been subjected to the full process of purification and will therefore take up the liquid crystalline state, has a filtration end-point greater than $450 \mathrm{~m} \mu$; it is clear, therefore, that intermediate stages of aggregation are possible.

\title{
Origin of the Solar System
}

$\mathrm{T}$ HE presidential address to the British Astronomical Association was delivered by Rev. Dr. M. Davidson, on October 26, at Zion College, and dealt with the theories advanced to explain the system of planets and satellites of the solar system.

After brief reference to certain electric theories, in particular to that of H. P. Berlage, which appeared in Prof. Roy. Acad. Amsterdam in 1930-34, other theories which have held the field for a time were discussed. The main objection to the old and rather attractive Laplacian hypothesis is the enormous discrepancy between the angular momentum that the rotating nebula must have possessed and that which the solar system possesses to-day. Further, the theory suggested that the planets were thrown off from the original sun by internal forces and were not removed by some outside agency; but, as the major planets have about 98 per cent of the angular momentum of the solar system and only about 1/700 of the total mass, this suggests the action of an internal body.

The planetismal hypothesis of Chamberlin and Moulton did not receive so much attention in Great Britain as might have been expected. The manner in which the planets were formed, according to this hypothesis, differs essentially from that which the more recent tidal theory assumes. In the former case, they are assumed to have been built up slowly by accretions drawn into the original nuclei; in the latter case the once liquid planets received very little later accretions. The difference may not be of much interest to an astronomer but to the geologist it is of the utmost importance.

The planetismal hypothesis is the parent of the more recent tidal theories, and naturally many of the objections to the latter will also apply to the former. Dealing with Sir James Jeans's theory, which first assumed a very extensive sun but later adopted the 'Roche model'--a body with a strong central con.
densation-Dr. Davidson referred to a number of objections to the theory. The greatest of these, he believes, is that which arises from a consideration of the distribution of angular momentum in the planets, per unit mass, as pointed out by Prof. H. N. Russell. The visiting star which was supposed to have produced the eruption from our sun must have made a very close approach, and, even under the most favourable conditions, it could not have produced ten per cent of the angular momentum per unit mass that the planets possess at present. This seems to be sufficient, without any other objections, to condemn the theory.

The recent theory of Dr. R. A. Lyttleton was then dealt with. This starts with the assumption that the sun was once a binary and that its companion was ejected by a visiting star, the filament formed by the usual disruption, which is supposed to occur in these cases, finally producing the planets and satellites, the latter by close approaches of planets. However, as Dr. H. Jeffreys has shown, the satellites could not have been formed in the manner assumed, and $\mathrm{Dr}$. Lyttleton was forced to abandon his original explanation of these bodies, and to assume rotational instability of enormous primitive planets. Jupiter and Saturn, according to this theory, were once a single extensive planet, and from the filament resulting from the disruption their satellites were formed, and even other independent planets. His explanation of the origin of the primitive planets was severely criticized by Dr. W. J. Luyten and Dr. E. L. Hill. In particular, they had shown that the visiting star, which must have removed the filament from the companion at least 20 astronomical units from the sun (half the distance of Pluto), required a velocity of about $100 \mathrm{~km}$. a second, and at such a distance from the sun the matter would escape. There are other objections, and Dr. Lyttleton, recognizing the validity of these, has modified the 
theory in one respect especially. If the visiting star is greater than the companion of the sun, the conditions would obviously be more favourable, but, on the whole, it seems extremely doubtful if an expla. nation will be found along these lines. Dr. Davidson is of opinion that some much simpler explanation, not demanding so many ad hoc assumptions, would be forthcoming, but this might be a long way off yet.

In conclusion, Dr. Davidson referred to the new satellites of Jupiter and to the fact that Mr. J. Miller had predicted a missing satellite (NATURE, Feb. 5, p. 245). Unfortunately, satellite $\mathrm{x}$, for which an orbit had been computed, did not fit into the place predicted for it, as it was 7 million miles from Jupiter. He felt, however, that there was a space about $2 \frac{1}{2}$ million miles from Jupiter in which a satellite would some day be discovered. The fact that satellites vi, vii, and $x$ are moving in similar orbits at nearly the same mean distances from their primary provides another problem for the cosmogonist. Perhaps a shoal of minute bodies occupies this region, but naturally one can only conjecture on such matters.

At the close of the address, Dr. Davidson vacated the presidential chair, which was then taken by $\mathrm{Mr}$. B. M. Peek, the newly elected president.

\section{Removal of Sulphur Acids from Flue Gases}

\begin{abstract}
T Great Britain there is manufactured annually about one million tons of sulphuric acid, but a quantity of sulphur oxides much greater than this is discharged into the atmosphere by the combustion of coal. In the last ten years, it has become possible to reduce atmospheric pollution from this source. The concentration of sulphur oxides in chimney gases is very low and so long as fuel-consuming units were small and scattered, the resulting damage was relatively inconspicuous. Moreover, any effective process for desulphurizing the gas would have been prohibitive in cost. Engineers limited their efforts to reducing the visible pollution by smoke and grit.
\end{abstract}

Technical developments in the generation of electricity compelled attention to the problem of cleaning flue gas. Mechanical stoking made it possible to burn fine coal at high rates of combustion and caused the discharge of much grit and dust into the atmosphere. The introduction of pulverized fuel accentuated the grit nuisance. Economy of steam production and power generation was secured by working in ever larger units and the need for reducing the high cost of distributing electricity required that these units should be installed near the load. The result has been the erection of huge coal burning units using inferior coal in large centres of population. With the project to erect the generating station at Battersea, the danger of discharging large quantities of sulphur acids into the heart of London was recognized, and the London Power Company was compelled to render its chimney gases innocuous before passing into the air. Now the concentration of coal burning into large units, while increasing the local intensity of atmospheric pollution, eased the technical problem of designing and operating economic processes of sulphur removal. In a relatively short time, the Battersea station was discharging into the air, flue gases almost free from sulphur. The gases, before entering the chimney, were washed with large volumes of Thames water to which lime was added and the clarified effluent returned to the river.

When the Fulham Power Station came to be enlarged, the authorities were able to insist that a similar standard of purity should be maintained; but in addition, that no effluent should be returned to the Thames, lest the alkalinity of the river water, already diminished by the Battersea plant, should be destroyed. The very existence of the Fulham plant was thus dependent on devising a process which made no liquid effluent. The wash liquor would inevitably become saturated with salts which must be removed as fast as made. The salt in this case was calcium sulphate, which is prone to form a troublesome scale on surfaces and in a continuous process liable to block the plant.

In an address delivered at a recent meeting of the London Section of the Society of Chemical Industry, Dr. R. Lessing described the course of experiments which showed how the salt could be removed without scale formation. Calcium sulphate solutions can persist in a super-saturated state; but this condition can be prevented if the wash liquor contains sufficient $\mathrm{CaSO}_{4} \cdot 2 \mathrm{H}_{2} \mathrm{O}$ on suspension. It was found possible to define conditions under which the flue gases could be washed continuously with a liquor, containing in suspension about 10 per cent of $\mathrm{CaSO}_{4}$ and working in a closed cycle. Water removed by evaporation up the chimney was replaced and fresh lime was added to replace that removed by precipitation of lime salts. An essential feature in the cycle was a 'delay' tank where the 'desupersaturation' of the liquor could proceed to a point where deposition of scale in the plant would not occur.

This process has now been in operation at Fulham and elsewhere long enough to leave no doubt that gases can be discharged from chimneys practically free from sulphur acids without the production of a liquid effluent. An important advantage of a washing process is the simultaneous removal of grit. Mechanical methods of removing grit are only partially successful. The electrostatic precipitator can retain grit almost completely, but allows acid gases to pass into the air. Moreover, the electrostatic precipitator is not certain under overload conditions such as arise when "soot blowing" is in progress.

The cost of sulphur extraction during the first year's working at Fulham was $0.0144 d$. per unit, and will doubtless be reduced with further experience. This may be a price well worth paying to reduce the damage due to sulphur in urban atmosphere. Dr. Lessing said that the washing of the chimney gases at Battersea and Fulham would eventually mean a reduction of sulphur pollution in the London area amounting to ten per cent. This shows the importance of this striking success of chemical engineering.

H. J. HodsmaN. 